\title{
Intensity-Modulated Radiotherapy Inverse Planning
}

National Cancer Institute

\section{Source}

National Cancer Institute. Intensity-Modulated Radiotherapy Inverse Planning. NCI

Thesaurus. Code C152033.

A procedure that utilizes a computerized algorithm to directly determine aperture

intensities for optimal radiation dose distributions associated with intensity-modulated radiotherapy (IMRT). 